\title{
Internet y territorio, o cuando la Sociedad de la Información cristaliza en desigualdad
}

\author{
Internet and territory, or when the Information Society crystallizes in \\ inequality
}

\author{
Sara MANDIÁ-RUBAL \\ Universidade de Santiago de Compostela, USC (España) \\ sara.mandia.rubal@gmail.com \\ https://orcid.org/0000-0003-4452-2751
}

Recibido: 22 de febrero del 2019

Aceptado: 30 de marzo 2019

\section{Resumen}

La Sociedad de la Información ha sido entendida de muy diversos modos. Las dos posiciones más claras se dividen entre los que la ven como una herramienta necesaria para la superación de la distancia, y aquellos que opinan que el espacio sigue siendo un problema, y que se acentúa año a año la brecha entre quienes se sitúan del lado de la digitalización y los que no. La hipótesis que se plantea es si la Sociedad de la Información cristaliza la desigualdad preexistente. Para poder obtener los datos con los que realizar las comparativas, se toma como referencia la fórmula del International Telecommunication Union, el IDI o Índice de Desarrollo de las TIC. Como resultado, se puede decir que la Sociedad de la Información cristaliza la desigualdad. Siempre son los mismos países los que se encuentran en las mejores y peores posiciones en cuanto al uso y explotación de las TIC. Trece países ocupan siempre el Top Ten, y más del $60 \%$ de esos trece países son europeos. Los últimos tres puestos son siempre para países africanos.

\section{Abstract}

The Information Society has been understood in many ways. The two clearest positions are divided between those who see it as a necessary tool for overcoming distance, and those 
who believe that space continues to be a problem, and that the gap between those who are on the side is accentuated year after year of digitization and those that are not. The hypothesis that arises is whether the Information Society crystallizes in a pre-existing inequality. For the calculations, the ICT Development Index (IDI) of the ITU, International Telecommunication Union is taken as a reference. As a result, it can be said that the Information Society crystallizes inequality. It is always the same countries that are in the best and worst positions in terms of the use and exploitation of ICTs. Thirteen countries always occupy the Top Ten, and more than $60 \%$ of those thirteen countries are European. The last three positions are always for African countries.

Palabras Clave: desigualdad; globalización; Internet; Sociedad de la Información; Tecnologías de la Información y la Comunicación (TIC); territorio.

Keywords: globalization; inequality; Information and Communication Technologies (ICT); Internet; Society of Information; territory.

\section{Introducción}

La Sociedad de la Información ha sido entendida de muy diversos modos. Las dos posiciones más claras se dividen entre los que la ven como una herramienta necesaria para la superación de la distancia, por las posibilidades que ofrecen las Tecnologías de la Información y la Comunicación (TICs); y aquellos que opinan que el espacio sigue siendo un problema, y que se acentúa año a año la brecha entre quienes se sitúan del lado de la digitalización y los que no.

Juzga Torres-Enjuto (2003) que las telecomunicaciones son las infraestructuras que soportan los servicios asociados a la Sociedad de la Información "que tiene como buque insignia Internet” (p. 160), y la destrucción de estas redes lógicas por el territorio es muy dispar, tanto en tipología, como en capacidad, y localización.

La información precisa de una nueva infraestructura de comunicaciones, y en la Sociedad de la Información las telecomunicaciones son el soporte técnico esencial de esta nueva era. Las 
telecomunicaciones son la nueva infraestructura capaz de transmitir la información en sus diferentes formas (texto, imágenes, voz) a una enorme velocidad y a un coste más que aceptable. Mientras que hasta hace unos años las tarifas de las telecomunicaciones sólo estaban al alcance de las empresas multinacionales, la difusión de Internet ha significado el inicio de un uso generalizado por empresas y ciudadanos. No obstante, la distribución de las infraestructuras de telecomunicaciones dista bastante de ser homogénea en el territorio. Las infraestructuras y servicios de telecomunicaciones que permiten los usos más sofisticados, cuyos clientes son empresas y organismos diversos, están localizados en las metrópolis (Torres-Enjuto, 2003, p. 158).

Para Castells (2002) estamos ante una "nueva economía", caracterizada por el uso de la información, "la productividad y la competencia se basan en el conocimiento y la información, impulsados por la tecnología de la información" (p. 93); la conexión en red, "el aspecto negativo de esta dinámica es que el sistema permite la conexión y desconexión de regiones, e incluso de países, en función de su contribución a la cadena de valor estructurada en torno a esas redes globales" (p. 93); y el marco de una economía global, "cuyas actividades fundamentales tienen la capacidad de trabajar como una unidad en tiempo real y a escala planetaria" (p. 93).

Las reglas de este nuevo orden económico, son para el autor (Castells, 2002, p. 94): la preponderancia de Internet; una mano de obra "altamente especializada, flexible, adaptable, autoprogramable, capaz de innovar"; y los mercados como "núcleo de la realización de valor". El propio autor, comenta que,

La revolución de las tecnologías de la información lo cambia todo. En los actuales parámetros de división internacional del trabajo, los países y las regiones pobres están amenazados con una irrelevancia estructural, asociada a su obsolescencia tecnológica. (...) El conocimiento y la información son las claves de la productividad, la conectividad es la 
clave de la competencia global. (...) La creación de una infraestructura de Internet requiere la renovación y extensión del sistema de telecomunicaciones (Castells, 2002, p. 99).

"La economía global está fuertemente segmentada: no todos están incluidos, pero todos resultan afectados" (Castells, 2002, p. 94). Hoy la digitalización está en el centro de todos los procesos que afectan a la vida del ciudadano: en la forma de comunicar e interaccionar, con las 'Information and Communication Technologies'; en la producción de bienes y servicios, con el 'internet of things'; en lo económico, con el 'e-commerce'; en lo financiero, con la 'electronic banking service'; en la política y la administración, con la 'e-administration'; en la enseñanza, y la 'e-learning'; etcétera.

Parece que Internet y la Sociedad de la Información han traído consigo una nueva desigualdad: la digital.

La investigación que a continuación se detalla pretende, precisamente, dar respuesta a la siguiente afirmación hipotética: la Sociedad de la Información cristaliza la desigualdad preexistente.

\section{Estado de la Cuestión}

En la actual concepción del mundo, la distribución de la inteligencia —riqueza, en última instancia- depende de factores extrínsecos al propio Ser Humano.

Factores como localización, condición personal o entorno cultural, determinan hoy la posición y rol de cada cual en la Sociedad de la Información. Esta idea es coincidente con la expuesta por Copus (2008) cuando afirma el "fin de la periferia espacial clásica" para ver nacer un nuevo tipo de periferia: la social, presente en todo el mundo, independientemente de situarse del lado ventajoso o desventajoso del desarrollismo económico. 
Mientras que para González Sánchez (2003, p.11) "Cultura de la Información, Cultura de la Investigación y Cultura de la Comunicación" son tres ejes que determinan hoy el desarrollo futuro de una sociedad, Copus (2008) prefiere hablar de "accesibilidad" y "perifericidad".

González Sánchez (2003) entiende que el mundo se ha dividido en dos colectivos: los "autoprogramables", generadores del conocimiento necesario para progresar, precisamente por ser ellos los que cuenten con la posibilidad de acceder a esas tres formas de cultura, gracias a las herramientas tecnológicas que poseen; y los "programados", que asumen ese nuevo conocimiento, así como el rol asignado — de producir y consumir-.

Para Copus (2008), en una conceptualización más clásica del problema, "accesibilidad" depende de un conjunto de elementos que pueden clasificarse en: elementos causales, elevados costos de transporte; elementos contingentes, bajas tasas de innovación e iniciativa empresarial; y elementos asociados, como la existencia de población dispersa, dependencia de actividades económicas primarias, o deficientes infraestructuras locales.

Con "periferia no espacial" o "aespacial” se refiere el autor (Copus, 2008) a una visión más moderna del problema, definida por la ausencia de nuevas tecnologías de la información y la comunicación, tanto en lo que se refiere a las infraestructuras disponibles como en el uso que se hace de ellas; innovación y redes empresariales; capital social; gobierno del territorio -planificación e intervención-; y grado de integración con las redes globales, principalmente en lo referente a la actividad turística.

En todo proceso de modernización, el cambio genera una nueva estructura de oportunidades, pero, conjuntamente, también cabe la posibilidad de que aparezcan nuevos tipos de desigualdad social o de que, al menos, se acentúen las ya existentes. La transición hacia la Sociedad de la Información no es una excepción. (...) Estas desigualdades, referidas al acceso a la información y al conocimiento 
mediante las nuevas tecnologías que lo facilitan (las TIC) se recogen bajo el concepto de brecha digital (Robles y Molina, 2007, p. 81).

La citada brecha digital es precisamente ese agravio comparativo, entre quienes se sitúan dentro de la Sociedad de la Información y los que no. Afecta tanto a economías industriales y post-industriales avanzadas, como a las zonas menos desarrolladas del planeta (Malecki, 2003), variando simplemente el factor de influencia para situarse de un lado u otro: países desarrollados vs países en vías de desarrollo - factor de localización-; ricos vs pobres —condición personal—; periferias espaciales o medio rural vs centro o metrópolis —entorno cultural-.

Las consecuencias son sin embargo las mismas, independientemente del factor que afecte. Aquellos que se sitúan del lado desventajoso permanecen condenados a la marginalidad en decisiones e inversiones, hasta que los aventajados decidan ayudarlos (Mandiá-Rubal, 2019, p. 91).

Hasta no hace mucho tiempo las redes de telecomunicaciones eran como la electricidad, el agua u otros servicios básicos, inversiones necesariamente públicas por ser de interés general y tener carácter estratégico, aunque no necesariamente rentables. Este es el caso de Telefónica S.A. en España, empresa mayoritariamente pública en su momento, cuya privatización total se acomete en 1995 y 1999 mediante dos ofertas públicas de acciones.

Las inversiones estratégicas no son necesariamente rentables por sí mismas, pero se entiende que atraen la inversión y resultan beneficiosas para el conjunto de la población a la larga. Internet, baluarte por excelencia de la Sociedad de la Información es, o debería ser, uno de esos servicios públicos necesarios.

En la era de la información, la matriz de todo desarrollo (tecnológico, económico, social) está en la innovación, en el valor supremo de la innovación que, potenciada por la revolución tecnológica informacional, incrementa exponencialmente la 
capacidad de generación de riqueza y de acumulación de poder (Castells, 2003, p. 12).

Para Malecki (2003), una buena planificación de la inversión pública debería tener presente que la instalación de empresas con una cierta base tecnológica no hace sino atraer la inversión de los monopolios que proveen de infraestructuras, actuando así el sector público como resorte del cambio modernizador.

Una regla de oro para el atractivo de un área, para la agregación de transmisión de datos, es el número de ordenadores por unidad de área. Las zonas rurales tienen que demostrar que tienen suficiente demanda para ser un mercado atractivo (Malecki, 2003, p. 210).

Pareciera que, si bien Internet permite "la muerte de la distancia" (Torres Enjuto, 2003) y un "aplanamiento del terreno de juego" (Friedman, 2006) que es el Planeta, ya la imprenta posibilitó el flujo de información y sin embargo, solo unos pocos hubieron podido históricamente acceder a una cantidad suficientemente importante de conocimiento como para poder innovar y probar nuevas formas de progreso.

Es complicado alcanzar un sistema más igualitario y democrático en la distribución de roles sociales y grado de bienestar, porque el sistema actual cristaliza y se eterniza en una doble desigualdad: la desigualdad de acceso a los soportes materiales para generar conocimiento; y la ausencia de disposiciones cognitivas para construir, asimilar y aprovechar esas tecnologías e infraestructuras por ciertas capas sociales.

A pesar de que la Red es una herramienta relativamente sencilla para acercar al conocimiento, todos por igual, y garantizar que al menos posean las herramientas necesarias para modificar su destino, lo cierto es que esta tendencia verticalizarte se muestra difícilmente modificable (González Sánchez, 2003). 


\section{Objetivos, Hipótesis y Metodología}

Internet y la Sociedad de la Información han traído consigo una nueva desigualdad. La digital. Cuando además la digitalización está en el centro de todos los procesos que afectan al ciudadano: en la forma de comunicar e interaccionar, con las 'Information and Communication Technologies'; en la producción de bienes y servicios, con el 'internet of things'; en lo económico, con el 'e-commerce'; en lo financiero, con la 'electronic banking service'; en la política y la administración, con la 'e-administration'; en la enseñanza, y la 'elearning'; etcétera.

Con este objetivo nace esta investigación. Afirmar o refutar, con datos, la siguiente afirmación —hipótesis de investigación-: la Sociedad de la Información cristaliza la desigualdad preexistente.

Se sitúa, como objetivo secundario, la comparación entre México y España. Dos países de historia compartida, situados a ambos lados del Atlántico.

Para poder obtener los datos con los que realizar las comparativas, se toma como medida la fórmula propuesta por la Unión Internacional de Telecomunicaciones, ITU en sus siglas inglesas. Un organismo especializado en telecomunicaciones de la Organización de las Naciones Unidas (ONU), con sede en Ginebra, encargado de regular las telecomunicaciones a nivel internacional entre las distintas administraciones y empresas operadoras.

Explica el citado organismo (ITU, 2019) que el IDI, o Índice de Desarrollo de las TIC, es un índice compuesto que combina once indicadores en una única unidad de medida; y se toma como referencia para monitorear y comparar la evolución de las tecnologías de la información y la comunicación (TIC) entre países, a lo largo del tiempo. En este caso desde 2009 que realizan su primera publicación con los datos de 2002 y 2007.

Los principales objetivos del IDI son: 
a) Medir el nivel de desarrollo de las TIC y su evolución a lo largo del tiempo, dentro de los países, y en relación a otros.

b) Medir el desarrollo de las TIC, tanto en países desarrollados como en vías de desarrollo.

c) Cuantificar la brecha digital. Esto es, la diferencia entre países en términos de desarrollo de las TIC.

d) Ponderar el potencial de desarrollo de las TIC y la medida en que los países pueden utilizarlas para mejorar su crecimiento, en el contexto de las capacidades y habilidades disponibles en la población.

El Índice está diseñado para ser global y reflejar los cambios que tienen lugar en países con diferentes niveles de desarrollo tecnológico. Se basa en un conjunto finito de datos que pueden establecerse con una confianza razonable en países de todos los niveles de desarrollo.

La selección de indicadores incluidos en el Índice de Desarrollo de las TIC se basa en, la relevancia del dato, la disponibilidad para obtenerlo, y la suma de resultados en la aplicación de varios análisis estadísticos —subíndices-.

En la Tabla 1 se recogen los once indicadores que están incluidos en el Índice de Desarrollo de las TIC (IDI) agrupados por tres subíndices: acceso, uso y habilidades.

Tabla 1: Indicadores incluidos en la IDI y distribución porcentual de cada uno de ellos en el cálculo final por país/año.

Subíndices Indicadores y porcentaje de peso en el cálculo anual por país

Acceso Suscripciones a telefonía fija por 100 habitantes

Suscripciones a telefonía móvil celular por cada 100 habitantes

Ancho de banda internacional de Internet (bit/segundo) por usuario de Internet

Porcentaje de hogares con computadora

Porcentaje de hogares con acceso a Internet

Uso

Porcentaje de personas que utilizan Internet
Distribución

$20 \%$

$20 \%$

$20 \%$

$20 \%$

$20 \%$

$33 \%$
$40 \%$

$40 \%$ 
Suscripciones de banda ancha fija por cada 100 habitantes 33\%

Suscripciones activas de banda ancha móvil por cada 100 habitantes $33 \%$

Habilidades Promedio de años de escolaridad

Tasa bruta de matrícula, nivel secundario

Tasa bruta de matrícula, nivel terciario

Fuente: Elaboración propia.

La normalización de los datos se realiza a través de una medida de referencia. Esa medida de referencia es el valor ideal —valor máximo/meta, para cada variable-.

Para todos los indicadores elegidos ese valor ideal es 100, excepto para los siguientes:

* Ancho de banda internacional de Internet, por usuario de Internet. Para disminuir el efecto de la enorme dispersión previsible, los datos se convierten primero a escala logarítmica (log) y a continuación se identifican los valores atípicos utilizando un valor de corte calculado agregando dos desviaciones estándar a la media de valores reescalados.

* Suscripciones a celular móvil, por cada 100 habitantes. Se revisa cada año y se establece en función de los datos objetivos.

* Abonos a telefonía fija, por 100 habitantes. El valor de referencia se calcula sumando dos desviaciones estándar a la media, dando como resultado un valor redondeado.

* Suscripciones de banda ancha fija, por cada 100 habitantes. En línea con las suscripciones de telefonía fija, el valor ideal se redefine periódicamente y da como resultado un valor redondeado.

* Años medios de escolaridad. Para este indicador se utiliza el valor ideal de referencia, que de nuevo no es 100 sino 15. Entendiendo que 15 son los años máximos de escolaridad necesarios para alcanzar un título universitario o un grado superior en formación profesional -en circunstancias normales el alumno entraría al mercado laboral, con capacitación, con 21-22 años de edad-.

Atendidas estas particularidades, y explicado el método cuantitativo aplicado, se muestran a continuación los resultados y la comparativa entre países. 


\section{Resultados}

Tabla 2: ICT Development Index (IDI), 2002 - 2017. Top Ten, por país y año.

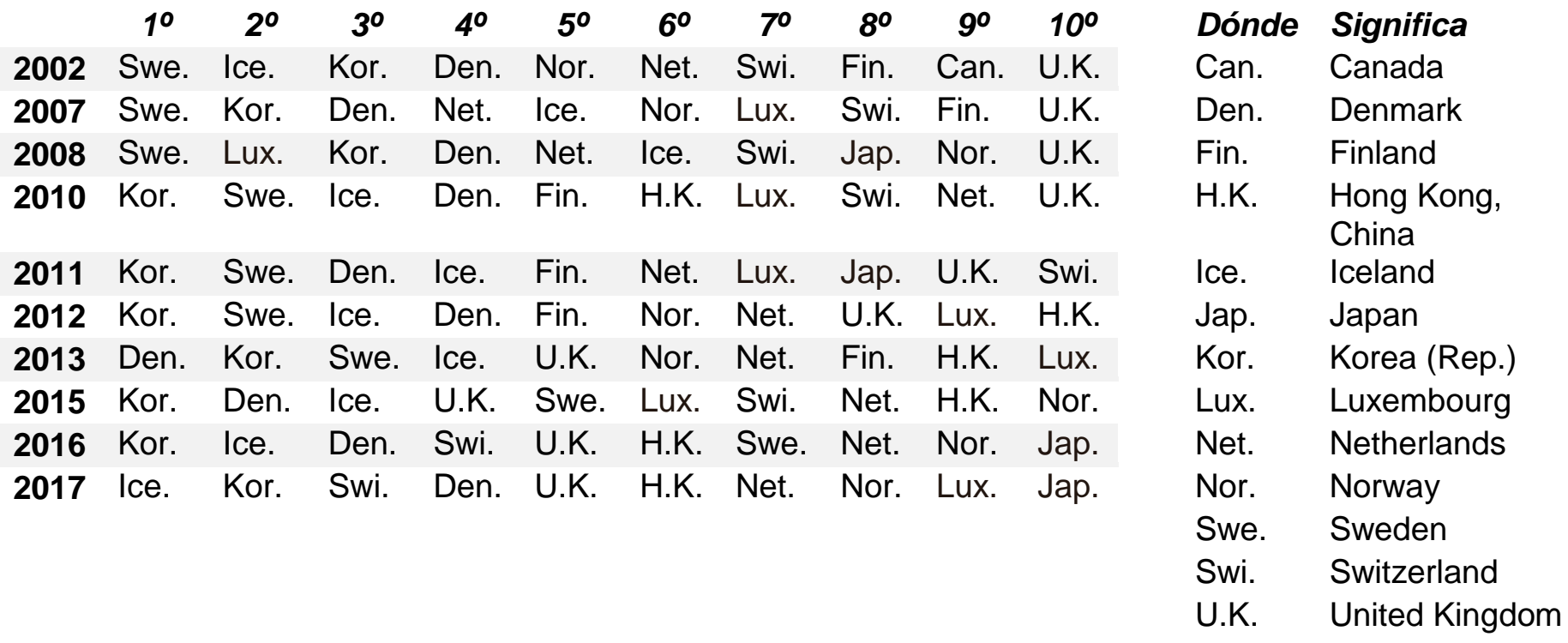

Fuente: Elaboración propia.

En toda la secuencia temporal, las diez primeras posiciones se reparten entre trece países -Tabla 2-.

Por orden alfabético, estos trece países serían: Canada, Denmark, Finland, Hong Kong, Iceland, Japan, Korea (Rep.), Luxembourg, Netherlands, Norway, Sweden, Switzerland, y United Kingdom.

Si el total muestreado en el IDI son alrededor de 170 países, estos trece primeros países representan el 7,6\% del total. Más del $60 \%$ del posible Top Ten son, año tras año, países del viejo continente.

En las tres últimas posiciones del ranking, por año, la Tabla 3 muestra que, en toda la secuencia temporal, las últimas posiciones siempre son ocupadas por estos siete países 
alternativamente: Central African Rep., Chad, Eritrea, Ethiopia, Guinea, Guinea-Bissau, Niger — de nuevo listados por orden alfabético-.

Tabla 3: ICT Development Index (IDI), 2002 - 2017. Tres últimos países del ranking por año.

\begin{tabular}{|c|c|c|c|}
\hline & Último & Penúltimo & Antepenúltimo \\
\hline 2002 & Niger & Guinea-Bissau & Chad \\
\hline 2007 & Niger & Chad & Guinea-Bissau \\
\hline 2008 & Chad & Niger & Guinea \\
\hline 2010 & Chad & Niger & Ethiopia \\
\hline 2011 & Niger & Chad & C.A.R. ${ }^{*}$ \\
\hline 2012 & Niger & C.A.R. ${ }^{*}$ & Chad \\
\hline 2013 & C.A.R. ${ }^{*}$ & Niger & Chad \\
\hline 2015 & Chad & Eritrea & Ethiopia \\
\hline 2016 & Niger & Chad & Guinea-Bissau \\
\hline 017 & Chad & C.A.R. ${ }^{*}$ & Eritrea \\
\hline
\end{tabular}

* Central African Republic.

Fuente: Elaboración propia.

Los siete son países del continente africano.

En una comparativa entre el primero y el último del ranking establecido por el ITU, la Unión Internacional de Telecomunicaciones de la ONU, se toman Korea y Chad por ser los que más veces se sitúan en ambos polos, el primero como número uno y el segundo más veces como último de la lista publicada por el organismo de la ONU -Tabla 4-.

Tabla 4: ICT Development Index (IDI), 2002 - 2017. Comparativa entre Korea y Chad.

\begin{tabular}{lrrrrrrrrrr} 
& $\mathbf{2 0 0 2}$ & $\mathbf{2 0 0 7}$ & $\mathbf{2 0 0 8}$ & $\mathbf{2 0 1 0}$ & $\mathbf{2 0 1 1}$ & $\mathbf{2 0 1 2}$ & $\mathbf{2 0 1 3}$ & $\mathbf{2 0 1 5}$ & $\mathbf{2 0 1 6}$ & $\mathbf{2 0 1 7}$ \\
Korea (Rep.) & 5,83 & 7,26 & 7,68 & 8,4 & 8,56 & 8,57 & 8,85 & 8,93 & 8,84 & 8,85 \\
Chad & 0,65 & 0,83 & 0,79 & 0,83 & 0,94 & 1,01 & 1,11 & 1,17 & 1,09 & 1,27 \\
& \multicolumn{8}{c}{ Fuente: Elaboración propia. }
\end{tabular}

Entre 2002 y 2017 Korea del Sur incrementa en más de tres puntos su IDI, o Índice de Desarrollo de las TIC. Mientras que Chad lo hace en un punto.

Apuntar, como curiosidad, que pese a las enormes diferencias que les separan, ambos países llegan a su punto álgido en 2015 , con 8,93 y 1,17 puntos respectivamente.

Entre ellos les separan 7,76 puntos, en un máximo de 8,93 que fija Korea ese año. 
Chad en el IDI de 2015, ocupa la última posición del ranking.

Gráfico 2: Representación gráfica de la Tabla 4.

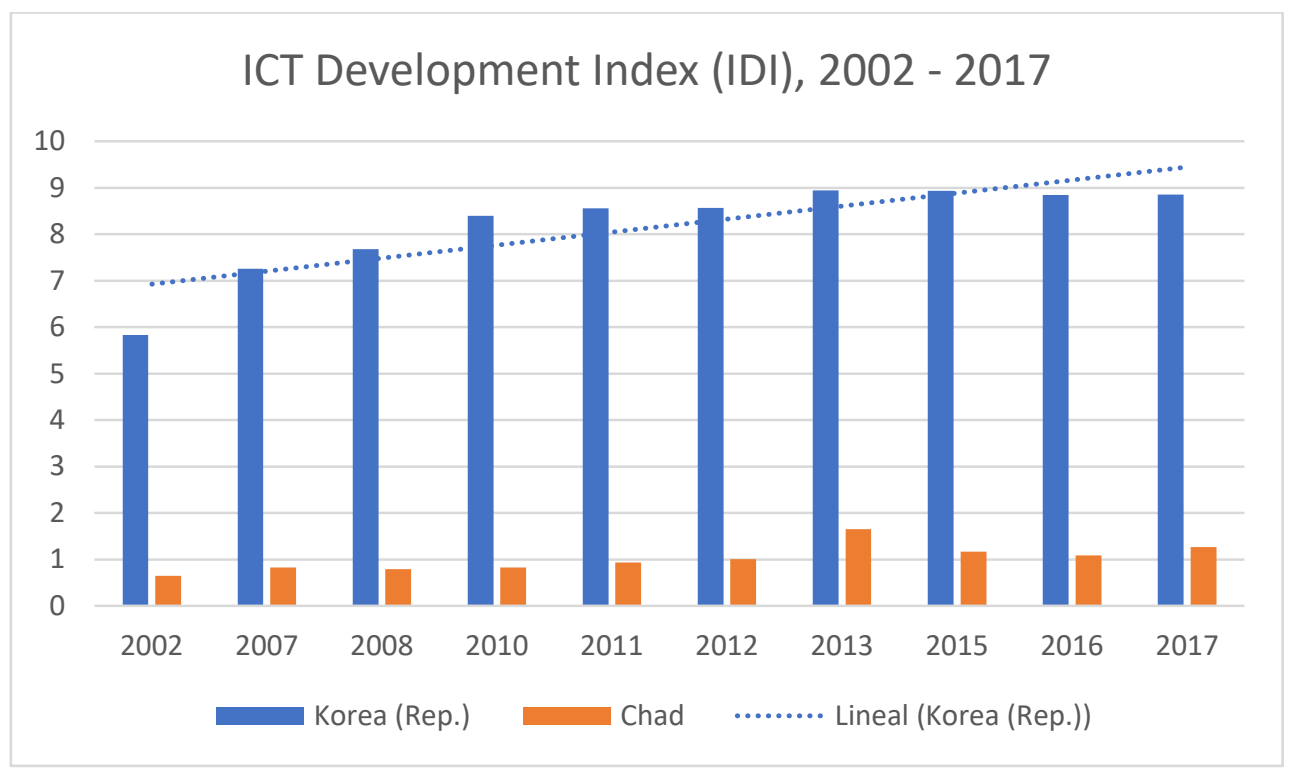

Fuente: Elaboración propia.

Se situaba como objetivo secundario, la comparación entre México y España. Dos países de historia compartida, situados a ambos lados del Atlántico.

La Tabla 6 establece esa comparativa, donde España crece a un ritmo mayor que México, también impulsada por los países de su entorno.

Veíamos anteriormente que más del $60 \%$ de los países que tradicionalmente ocupan las diez primeras posiciones del listado ITU son del viejo continente. No en vano la Unión Europea ha ayudado económica y políticamente a que así fuese, con estímulos y planes de desarrollo -tecnólogos, de digitalización, y de formación-.

Tabla 5: ICT Development Index (IDI), 2002 - 2017. Comparativa entre España y México.

$\begin{array}{lrrrrrrrrrr} & \mathbf{2 0 0 2} & \mathbf{2 0 0 7} & \mathbf{2 0 0 8} & \mathbf{2 0 1 0} & \mathbf{2 0 1 1} & \mathbf{2 0 1 2} & \mathbf{2 0 1 3} & \mathbf{2 0 1 5} & \mathbf{2 0 1 6} & \mathbf{2 0 1 7} \\ \text { Spain } & 4,1 & 5,91 & 6,27 & 6,73 & 6,62 & 6,89 & 7,38 & 7,66 & 7,62 & 7,79 \\ \text { México } & 2,38 & 3,09 & 3,25 & 3,75 & 3,74 & 3,95 & 4,29 & 4,68 & 4,87 & 5,16\end{array}$

Fuente: Elaboración propia. 
En este caso la puntuación más alta se alcanza en el último año del muestreo, con una secuencia continuada al alza, lo que hace pensar que ninguno de los dos países ha llegado todavía a su máximo, y que seguirán en la senda del aprovechamiento y uso eficiente de las TIC.

Entre 2002 y 2017 México ha mejorado en 2,78 puntos su total IDI. España lo ha hecho en 3,69 puntos.

En 2017 la diferencia entre ambos países era de 2,63 puntos.

\section{Gráfico 1: Representación gráfica de la Tabla 5.}

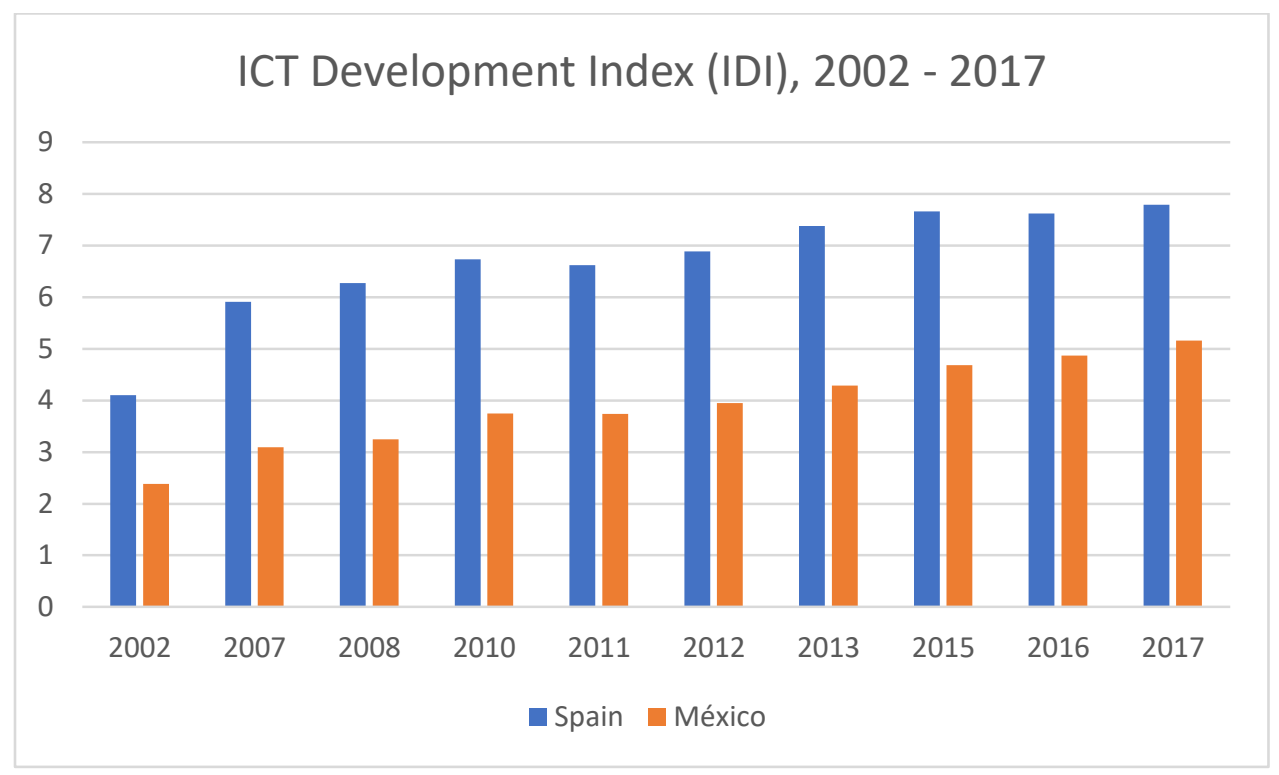

Fuente: Elaboración propia.

\section{Conclusiones}

Efectivamente, la Sociedad de la Información cristaliza la desigualdad preexistente.

Son siempre los mismos países los que se sitúan en las mejores y peores posiciones respecto al uso y aprovechamiento de las TIC. 
Trece países han ocupado el histórico Top Ten, y más del $60 \%$ de ellos pertenecen al continente europeo.

El $100 \%$ de los peor puntuados, son países del continente africano. Disputándose los tres últimos puestos del listado, año tras año, tan solo siete países; que en realidad se alternan, a poca distancia unos de otros, sin llegar a salir nunca de ese poco honorable registro.

Al primero y al último más frecuentes - Korea del Sur y Chad, respectivamente-, les separan 7,76 puntos; en unos máximos que no llegan al 9 en ninguna de las ediciones publicadas hasta el momento.

La financiación es el problema, la barrera inicial que niega a una parte del mundo el acceso a ese tren elitista de la vanguardia que es hoy la Red de redes.

Al respecto, Castells (2002), expresa qué

Es necesario romper mediante una acción deliberada el círculo vicioso actual entre la falta de condiciones para el desarrollo de la información en muchos países y regiones del mundo, y la dinámica de las redes, que incluyen todas las fuentes de valor y excluyen aquéllas que no tienen valor económico o tecnológico. Sólo una inyección de recursos y knowhow masiva, inmediata y coordinada puede invertir la dinámica actual, que está fragmentando el planeta (p. 104).

La tan cacareada ayuda internacional, que deberían facilitar las organizaciones supranacionales ya existentes, depende en última instancia de los países que ahora ocupan posiciones de dominio respecto de aquellos que se consideran potenciales receptores, y como tal cristaliza una cierta posición de ventaja, una desigualdad histórica.

Castells (2002) habla de un "Plan Marshall Tecnológico" que "al igual que hizo el Plan Marshall en Europa, este nuevo plan ayudaría a la reconstrucción del mundo, al tiempo que 
ayudaría a las empresas de los países donantes en sus intereses comerciales a largo plazo" (p. 104).

Gobiernos velando por la igualdad de comunidades; Estados legislando por la equidad entre regiones y territorios; y la Política con mayúsculas unida para incrementar sus recursos y capacidad de decisión, supervisando un reparto justo entre países que dibuje un mundo inclusivo agradable a todos los que habitan en él.

\section{Bibliografía}

Castells, M. (2002). Tecnologías de la información y la comunicación y desarrollo global. Revista de economía mundial, núm. 7, pp. 91-107.

Castells, M. (2003). Internet, libertad y sociedad: una perspectiva analítica. Polis: Revista Latinoamericana, núm. 4, pp. 1-26.

Copus, A. (2008). Capítulo1. Marco teórico-conceptual. Nuevos factores del desarrollo territorial. [Valencia, España]: Universitat de València, pp. 31-57.

Friedman, T. (2006). La Tierra es Plana: breve historia del mundo globalizado del siglo XXI. Madrid, España: Martínez Roca.

García-Canclini, N. (2000). Industrias Culturales y globalización: procesos de desarrollo e interacción en América Latina. Estudios Internacionales, vol. 33, núm. 129, pp. 90-111.

González-Sánchez, J.A. (2003). Redes y sistemas de información (o el sueño de Prometeo sin cadenas). Cultura(s) y Cibercultur@(s). Incursiones no lineales entre complejidad y comunicación. [México DF, México]: Universidad Iberoamericana.

ITU, International Telecommunication Union (2009). “Table 4.2: ICT Development Index (IDI) (2002 and 2007)". En Measuring the Information Society Report 2009, pp. 32. Recuperado de https://www.itu.int/en/ITU-D/Statistics/Pages/publications/mis2009.aspx 
ITU, International Telecommunication Union (2010). "Table 2.2: ICT Development Index (IDI), 2008 and 2007". En Measuring the Information Society Report 2010, pp. 24. Recuperado de https://www.itu.int/en/ITU-D/Statistics/Pages/publications/mis2010.aspx

ITU, International Telecommunication Union (2011). "Table 2.2: ICT Development Index (IDI), 2010 and 2008". En Measuring the Information Society Report 2011, pp. 27. Recuperado de https://www.itu.int/en/ITU-D/Statistics/Pages/publications/mis2011.aspx

ITU, International Telecommunication Union (2012). "Table 2.2: ICT Development Index (IDI), 2010 and 2011". En Measuring the Information Society Report 2012, pp. 37. Recuperado de https://www.itu.int/en/ITU-D/Statistics/Pages/publications/mis2012.aspx

ITU, International Telecommunication Union (2013). "Table 2.2: ICT Development Index (IDI), 2011 and 2012". En Measuring the Information Society Report 2013, pp. 42. Recuperado de https://www.itu.int/en/ITU-D/Statistics/Pages/publications/mis2013.aspx

ITU, International Telecommunication Union (2014). "Table 2.2: ICT Development Index (IDI), 2012 and 2013". En Measuring the Information Society Report 2014, pp. 60. Recuperado de https://www.itu.int/en/ITU-D/Statistics/Pages/publications/mis2014.aspx

ITU, International Telecommunication Union (2015). "Table 2.2: IDI overall rankings and ratings, 2015 and 2010". En Measuring the Information Society Report 2015, pp. 62. Recuperado de https://www.itu.int/en/ITU-D/Statistics/Pages/publications/mis2015.aspx

ITU, International Telecommunication Union (2016). "Table 1.7: IDI value change, 2015-2016". En Measuring the Information Society Report 2016, pp. 40. Recuperado de https://www.itu.int/en/ITU-D/Statistics/Pages/publications/mis2016.aspx

ITU, International Telecommunication Union (2017). "Table 2.2: IDI rankings and values, 2017 and 2016". En Measuring the Information Society Report 2017, pp. 45. Recuperado de https://www.itu.int/en/ITU-D/Statistics/Pages/publications/mis2017.aspx 
ITU, International Telecommunication Union (2019). The ICT Development Index (IDI): conceptual framework and methodology. Recuperado de https://www.itu.int/en/ITUD/Statistics/Pages/publications/mis/methodology.aspx

Malecki, E. (2003). Digital development in rural areas: potentials and pitfalls. Journal of Rural Studies, vol. 19, núm. 2, pp. 201-214.

Mandiá-Rubal, S. (2019). "El Conocimiento en línea: un interesante eslabón de la apropiación tecnológica". En Experiencias innovadoras educativas, pp. 89-112. Baja California: UABC Universidad Autónoma de Baja California.

Miguel, J.C. (2000). Industrias culturales: gratuidad y precios en Internet. ZER Revista de estudios de comunicación, vol. 5, pp. 115-150.

Robles, J.M., \& Molina, O. (2007). La brecha digital: ¿una consecuencia más de las desigualdades sociales? Un análisis de caso para Andalucía. Empiria: Revista de metodología de ciencias sociales, núm. 13, pp. 81-100.

Torres-Enjuto, M.C. (2003). La geografía de la sociedad de la información: ¿real o virtual?. Boletín de la Asociación de Geógrafos Españoles, vol. 35, pp. 153-171. 